\title{
Received Probability of Vortex Modes Carried by Localized Wave of Bessel-Gaussian Amplitude Envelope in Turbulent Seawater
}

\author{
Shibao Deng, Yun Zhu and Yixin Zhang * \\ School of Science, Jiangnan University, Wuxi 214122, China \\ * Correspondence: zyx@jiangnan.edu.cn
}

Received: 30 April 2019; Accepted: 28 June 2019; Published: 30 June 2019

\begin{abstract}
By using the two-frequency coherence function model of a beam in a turbulent medium and the localized wave theory of the polychromatic beam, we develop the spectrum average mutual coherence function of the localized wave of Bessel-Gaussian amplitude envelope and the spectrum average coherence length of spherical wave. By the spectrum average coherence length and the spectrum average mutual coherence function, we construct a received probability of vortex modes carried by localized wave of Bessel-Gaussian amplitude envelope in anisotropic turbulent seawater. Our results show that the received probability of signal vortex modes increases with the increase of half-modulated pulse width of the input pulse, turbulent inner scale, anisotropic factor of turbulence and rate of dissipation of kinetic energy per unit mass of fluid, but it increases with the decrease of the Bessel cone angle and the dissipation rate of the mean-squared temperature. We also find that there is a maximum effective beam waist for a given receiving aperture, and the vortex mode is more sensitive to salinity fluctuations than to temperature fluctuations in turbulence. Our conclusions show that localized wave of Bessel-Gaussian amplitude envelope is a more suitable beam for the vortex mode communication than conventional vortex waves.
\end{abstract}

Keywords: localized wave of Bessel-Gaussian amplitude envelope; spectrum average mutual coherence function; spectrum average coherence length; vortex modes; seawater turbulence

\section{Introduction}

The development of marine wireless optical communications is of great significance in remote submersibles, submarines, analysis of marine resources, and exploration of undersea gas and oil. Therefore, the construction of an underwater high-speed, secure and high-capacity optical communication system is important. Because the use of vortex modes can achieve secure optical communication with high information content [1], research on the propagation of the vortex mode in turbulent media has gotten ferocious attention in the field of wireless optical communication [1-4]. However, when the vortex wave transits through turbulent seawater, it will suffer attenuation and wavefront distortion due to the various absorbing substance and the fluctuations of refractive index [5]. Considering that the vortex mode is determined by the wavefront structure, the wavefront deformation will generate mode distortion. It is noted that the wavefront deformation also includes phase distortion caused by optical systems [6], and "branch point" [7] contributed by the turbulent channel can also cause mode distortion. It is distortion of vortex mode that causes vortex crosstalk and vortex noise, which will reduce the quality of a wireless optical communication system with vortex modes. For this reason, a large number of studies have been reported about the propagation of vortex mode in turbulence of seawater [8-14]. For instance, Huang et al. [8] studied the evolution of Gaussian Schell model vortex beam in seawater turbulence. Liu et al. [9] analyzed the propagation of partially coherent 
flat-topped hollow beam carrying vortex mode in seawater turbulence. Zhao et al. [10] researched the evolution of four-fold vortex mode of green light $(520 \mathrm{~nm})$ in underwater channels of $2 \mathrm{~m}$ by experiments. Liu et al. [11] investigated the evolution of partially coherent four-petal Gaussian beam with vortex modes in seawater turbulence and found that when either decreasing the coherence length, the relative strength of the temperature and salinity fluctuations or the dissipation rate of kinetic energy per unit mass of fluid, or increasing the dissipation rate of the mean square temperature, the beam will soon be converted into a Gauss-like beam. Xu and Zhao [12] studied the propagation of a stochastic electromagnetic vortex beam in the turbulence of seawater, and found that a smaller topological charge corresponds to a smaller spectral degree of polarization.

Besides, a localized wave is one that has no diffraction for a long distance in the absence of turbulence [15]. For this reason, Ciattoni et al. [16] researched the transiting of vector electromagnetic localized wave in the absence of turbulence, and Ornigotti et al. [17] explored the propagation law of a new class of non-diffracting optical pulses with vortex mode. Recently, we investigated the received probability of vortex mode carried by an $\mathrm{X}$ wave in the turbulence of oceans. However, because the effect of frequency distribution is ignored in the calculation of the phase structure function [18], and the received probability function is a multiple integral, it is difficult to use this model to analyze problems such as bit error rate, channel capacity and so on. Therefore, by the Gaussian spectrum of the frequency of pulse, we superimpose the monochromatic Bessel-Gaussian beams to constitute the localized wave of Bessel-Gaussian amplitude envelope (BGLW) carrying vortex modes, and establish a new received probability of vortex modes that can be used to analyze signal bit error rate and the channel capacity of the communication link.

In this paper, for the BGLW with vortex modes in anisotropic seawater turbulence, we present a spectrum average mutual coherence function (SAMCF) by introducing a spectrum average coherence length of spherical wave. Using this SAMCF, we set up a more concise model of received probability of vortex modes carried by the BGLW in anisotropic seawater turbulence. With the help of the probability model, we discuss the influence of beam parameters, turbulence and receiver aperture on the received probability of vortex modes of the BGLW in weak seawater turbulence.

\section{Theoretical Model}

\subsection{Seawater Turbulence and Localized Wave of Bessel-Gaussian Amplitude Envelope}

In order to make the conclusions of this study closer to the real seawater environment, we research the propagation of BGLW with vortex modes in anisotropic turbulence. For the anisotropy of turbulent seawater that exists only along the $z$ direction of propagation, we have the power spectrum of the refractive index fluctuations [13]

$$
\phi^{\prime}\left(\kappa^{\prime}, \zeta\right)=0.388 C_{m}^{2} \zeta^{2} \kappa^{\prime-11 / 3}\left[1+2.35\left(\kappa^{\prime} \eta\right)^{2 / 3}\right] f\left(\kappa^{\prime}, \omega\right)
$$

where $\kappa^{\prime}=\sqrt{\kappa_{z}^{2}+\zeta^{2} \kappa_{\rho}^{2}}$ is the spatial frequency of the refractive index fluctuations, $\kappa_{\rho}=\sqrt{\kappa_{x}^{2}+\kappa_{y}^{2}}$, under the Markov approximation, $\kappa^{\prime}$ can be further simplified as $\kappa^{\prime}=\zeta \kappa_{\rho}, \zeta$ is the turbulence anisotropy index; $\omega$ is the ratio of temperature and salinity contributions to the refractive index spectrum, which can vary in $[-5 ; 0]$ in the seawater with -5 and 0 being heavily temperature and salinity dominating respectively, $\varepsilon$ is the dissipation rate of kinetic energy per unit mass of fluid that ranges from $10^{-10} \mathrm{~m}^{2} / \mathrm{s}^{3}$ to $10^{-1} \mathrm{~m}^{2} / \mathrm{s}^{3}, \chi_{T}$ is the dissipation rate of the mean-squared temperature and has the range from $10^{-10} \mathrm{~K}^{2} / \mathrm{s}$ to $10^{-4} \mathrm{~K}^{2} / \mathrm{s} ; f\left(\kappa^{\prime}, \omega\right)=\exp \left(-A_{T} \delta\right)+\omega^{-2} \exp \left(-A_{S} \delta\right)-$ $2 \omega^{-1} \exp \left(-A_{T S} \delta\right), \eta$ is the inner scale, $C_{m}^{2}=10^{-8} \mathcal{E}^{-1 / 3} \chi_{T}$ is the "temperature structure" constant and denotes the temperature fluctuation strength of seawater turbulence for given $\omega$ [19], $\delta=$ $8.284\left(\kappa^{\prime} \eta\right)^{4 / 3}+12.978\left(\kappa^{\prime} \eta\right)^{2}, A_{T}=1.863 \times 10^{-2}, A_{S}=1.9 \times 10^{-4}$, and $A_{T S}=9.41 \times 10^{-3}$. 
Using the Rytov approximation, we can express the complex amplitude of a Bessel Gaussian (BG) monochromatic vortex wave in turbulent seawater at the receiver plane as [20]

$$
u(\rho, \theta, z, \omega)=u_{m_{0}}(\rho, \theta, z, \omega) \exp [\mathrm{i} S(\rho, \theta, z, \omega)],
$$

where $(\rho, \theta, z)$ means cylindrical coordinates, $\omega$ is the angular frequency of the beam, $S(\rho, \theta, z, \omega)$ represents the phase perturbation of spectrum arising from seawater turbulence, $u_{m_{0}}(\rho, \theta, z, \omega)$ denotes the complex amplitude of the BG monochromatic vortex wave in the absence of turbulence and is expressed as $[18,21]$

$$
\begin{gathered}
u_{m_{0}}(\rho, \theta, z, \omega)=\frac{(-\mathrm{i})^{m_{0}+1} E_{0} \omega w_{0}^{2}}{2 z c \sqrt{1+z_{\xi}^{2}}} \exp \left[-\frac{\omega^{2} w_{0}^{2}}{4 c^{2}\left(1-\mathrm{i} z_{\xi}\right)}\left(\sin ^{2} \alpha+\frac{\rho^{2}}{z^{2}}\right)\right] \\
\times I_{m_{0}}\left(\frac{\omega^{2} w_{0}^{2} \rho \sin \alpha}{2 z c^{2}(1-\mathrm{i} z \xi)}\right) \exp \left[\mathrm{i} \frac{\omega}{c}\left(z+\frac{\rho^{2}}{2 z}\right)+\mathrm{i} m_{0} \theta+\mathrm{i} \arctan \left(z_{\xi}\right)\right],
\end{gathered}
$$

where $w_{0}$ is the beam waist, $I_{m_{0}}(\ldots)$ represents the modified Bessel function of the first kind, $m_{0}$ is the topological charge number of vortex mode, $\alpha$ is the Bessel cone angle, $E_{0}$ is a constant, $z_{\xi}=\omega w_{0}^{2} /(2 z c)$ is the non-dimensional coordinate. In Equation (3), we have changed the Bessel function of the first kind $J_{m}(\ldots)$ in $[18,21]$ to modified Bessel function of the first kind, through the following transformation [22]

$$
I_{m}(x)=\mathrm{i}^{-m} J_{m}(\mathrm{i} x),
$$

Applying the localized wave theory $[17,23]$ and using the frequency spectrum of the Gaussian pulse, we can express the localized wave of Bessel-Gaussian amplitude envelope (BGLW) transiting in turbulent seawater as

$$
u_{p}(\rho, \theta, z, t)=\frac{T_{0}}{2 \sqrt{\pi}} \exp (-\mathrm{i} \Omega t) \int_{-\infty}^{+\infty} \exp \left(-\frac{\omega^{2} T_{0}^{2}}{4}-\mathrm{i} \omega t\right) u(\rho, \theta, z ; \omega) \mathrm{d} \omega,
$$

where $T_{0}$ represents the half-modulated pulse width of the input pulse and $\Omega$ is center frequency.

Introducing new variables: the sum frequency $\omega_{c}=\left(\omega_{1}+\omega_{2}\right) / 2$ and the difference frequency $\omega_{d}=\omega_{1}-\omega_{2}$, in the case of narrowband, we have the approximate: $\omega_{c} \approx \widetilde{\Omega}$, where $\widetilde{\Omega}$ represents average frequency, meanwhile, $\omega_{c}$ relates to the location of the center frequency [24]. That is to say

$$
\Omega \approx \widetilde{\Omega}
$$

For the Gaussian spectrum of the frequency of pulse, we have

$$
\begin{aligned}
\widetilde{\Omega} & =\frac{\int_{0}^{\infty} \omega \exp \left(-\frac{T_{0}^{2}}{4} \omega^{2}\right) \mathrm{d} \omega}{\int_{0}^{\infty} \exp \left(-\frac{T_{0}^{2}}{4} \omega^{2}\right) \mathrm{d} \omega} \\
& =\frac{2}{T_{0} \sqrt{\pi}}
\end{aligned}
$$


2.2. Spectrum Average Mutual Coherence Function of Localized Wave of Bessel-Gaussian Amplitude Envelope

To obtain the received probability distribution of vortex modes for the BGLW, now we structure the spectrum average mutual coherence function (SAMCF) of BGLW in turbulent seawater. The SAMCF of output BGLW can be given by following expression $[20,23,25]$

$$
\begin{aligned}
\left\langle W\left(\rho, \theta_{1}, \theta_{2}, z ; t\right)\right\rangle & =\left\langle u_{p}\left(\rho, \theta_{1}, z ; t\right) u_{p}^{*}\left(\rho, \theta_{2}, z ; t\right)\right\rangle_{o c} \\
& =\frac{T_{0}^{2}}{4 \pi} \iint_{-\infty}^{+\infty} \exp \left[-\mathrm{i}\left(\omega_{1}-\omega_{2}\right) t\right] \Gamma_{2}\left(\rho, \theta_{1}, \theta_{2}, z ; \omega_{1}, \omega_{2}\right) \mathrm{d} \omega_{1} \mathrm{~d} \omega_{2}
\end{aligned}
$$

where $\langle\ldots\rangle_{o c}$ is the ensemble average of seawater turbulence, $\Gamma_{2}\left(\rho, \theta_{1}, \theta_{2}, z ; \omega_{1}, \omega_{2}\right)$ represents the two-frequency mutual coherence function (TFMCF) of BG vortex wave in seawater turbulence and should be written as

$$
\begin{aligned}
\Gamma_{2}\left(\rho, \theta_{1}, \theta_{2}, z ; \omega_{1}, \omega_{2}\right) & =\exp \left[-\frac{T_{0}^{2}}{4}\left(\omega_{1}^{2}+\omega_{2}^{2}\right)\right]\left\langle u\left(\rho, \theta_{1}, z, \omega_{1}\right) u^{*}\left(\rho, \theta_{2}, z, \omega_{2}\right)\right\rangle_{o c} \\
& =\Gamma_{2}^{0}\left(\rho, \theta_{1}, \theta_{2}, z ; \omega_{1}, \omega_{2}\right)\left\langle\exp \left[\mathrm{i} S\left(\rho, \theta_{1}, z, \omega_{1}\right)-\mathrm{i} S^{*}\left(\rho, \theta_{2}, z, \omega_{2}\right)\right]\right\rangle_{o c^{\prime}}
\end{aligned}
$$

where $\Gamma_{2}^{0}\left(\rho, \theta_{1}, \theta_{2}, z ; \omega_{1}, \omega_{2}\right)=\left\langle u_{m_{0}}\left(\rho, \theta_{1}, z, \omega_{1}\right) u_{m_{0}}^{*}\left(\rho, \theta_{2}, z, \omega_{2}\right)\right\rangle \exp \left[-\frac{T_{0}^{2}}{4}\left(\omega_{1}^{2}+\omega_{2}^{2}\right)\right]$ is the TFMCF of $B G$ vortex wave without turbulence interference.

\subsubsection{Spectrum Average Coherence Length of Spherical Wave}

Since localized wave is a kind of polychromatic wave, when we analyze the phase structure function, we consider the mean phase perturbation of spectrum $S(\rho, \theta, z, \widetilde{\Omega})$ [26] caused by seawater turbulence. For the Gaussian spectrum of the frequency of pulse, we have

$$
\begin{aligned}
S(\rho, \theta, z, \omega) & \approx S(\rho, \theta, z, \widetilde{\Omega}) \\
& =\frac{\widetilde{\Omega}}{c} \int_{0}^{z} n(\rho, \theta, z) \mathrm{d} z
\end{aligned}
$$

So we have the spectrum average structure function of the phase fluctuations

$$
\begin{aligned}
\left\langle\exp \left[\mathrm{i} S\left(\rho, \theta_{1}, z, \omega_{1}\right)-\mathrm{i} S^{*}\left(\rho, \theta_{2}, z, \omega_{2}\right)\right]\right\rangle_{o c} \approx & \left\langle\exp \left[\mathrm{i} S\left(\rho, \theta_{1}, z, \widetilde{\Omega}\right)-\mathrm{i} S^{*}\left(\rho, \theta_{2}, z, \widetilde{\Omega}\right)\right]\right\rangle_{o c} \\
= & \exp \left\{\frac{4 z \pi \zeta^{-4}}{3 T_{0}^{2} c^{2}} \int_{0}^{\infty} \kappa^{\prime 3} \phi^{\prime}\left(\kappa^{\prime}, \zeta\right) \mathrm{d} \kappa^{\prime}\right. \\
& \left.\times 2 \rho^{2}\left[\cos \left(\theta_{1}-\theta_{2}\right)-1\right]\right\} \\
= & \exp \left\{-\frac{2 \rho^{2}\left[1-\cos \left(\theta_{1}-\theta_{2}\right)\right]}{\rho_{o c \xi}^{2}}\right\} .
\end{aligned}
$$

where $\rho_{o c \zeta}$ represents the spectrum average coherence length of spherical wave transiting in the seawater turbulence and $\rho_{o c \zeta}^{-2}$ can be written as

$$
\rho_{o c \zeta}^{-2}=\frac{4 z \pi \zeta^{-4}}{3 T_{0}^{2} c^{2}} \int_{0}^{\infty} \kappa^{\prime 3} \phi\left(\kappa^{\prime}, \zeta\right) \mathrm{d} \kappa^{\prime}
$$


Similar to the discussion [19], we obtain the spectrum average coherence length of spherical wave in the anisotropic oceanic turbulence

$$
\rho_{o c \zeta}=\left[\frac{2.2937 \times 10^{-7} z(\varepsilon \eta)^{-1 / 3} \chi_{T}}{T_{0}^{2} c^{2} \zeta^{2}}\left(0.483-\frac{0.835}{\omega}+\frac{3.380}{\omega^{2}}\right)\right]^{-\frac{1}{2}} .
$$

Now, we plot the relationship between the spectrum average coherence length $\rho_{o c \xi}$ of spherical wave and the propagation distance $z$ under different "temperature structure" constant $C_{m}^{2}$ and different half-modulated pulse width $T_{0}$ in Figure $1 \mathrm{a}, \mathrm{b}$, respectively. There is no doubt that increasing the propagation distance or enhancing the turbulence strength can reduce the spectrum average coherence length [9-14]. From the expression of the coherence length, the parameter that we can control is the half-modulated pulse width $T_{0}$, and according to Figure $1 \mathrm{~b}$, increasing $T_{0}$ can effectively reduce the interference of oceanic turbulence on the spectrum average coherence length $\rho_{\text {oc } \xi}$ even in a strong turbulence environment. Therefore, increasing $T_{0}$ as often as possible is a good choice.

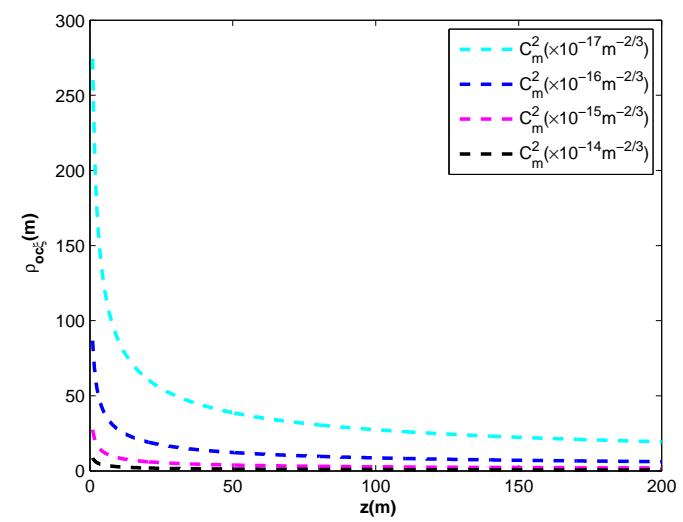

(a)

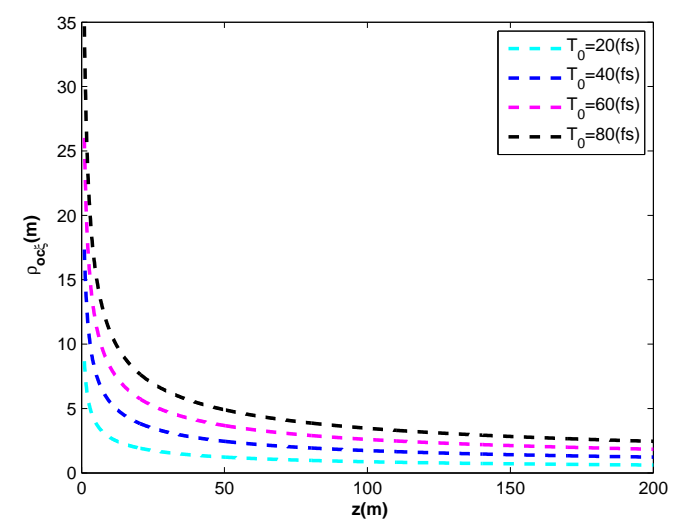

(b)

Figure 1. Spectrum average coherence length of spherical wave versus the propagation distance $z$ for different "temperature structure" constant $C_{m}^{2}$ under (a) $\omega=-4.5, T_{0}=20 \mathrm{fs}, \zeta=2$ and different half-modulated pulse width $T_{0}$ under $(\mathbf{b}) \omega=-4.5, C_{m}^{2}=10^{-14} \mathrm{~m}^{-2 / 3}, \zeta=2$.

\subsubsection{Spectrum Average Mutual Coherence Function}

By Equation (3), the approximation: $\omega w_{0}^{2} / 2 c=\pi w_{0}^{2} / \lambda \gg z$, the paraxial approximate and the approximate relationship: $I_{m_{0}}[x(1+\mathrm{i} y)] \sim \frac{[x(1+\mathrm{i} y) / 2]^{m_{0}}}{\Gamma\left(1+m_{0}\right)},(|x(1+\mathrm{i} y)| \ll 1)$, and by ignoring the turbulence effects, we can express the TFMCF of BG vortex beam as $[13,18]$

$$
\begin{aligned}
\Gamma_{2}^{0}\left(\rho, \theta_{1}, \theta_{2} ; \omega_{1}, \omega_{2}\right)= & E_{0}^{2} \pi T_{0}^{2} \exp \left[-2 \frac{z^{2}}{w_{0}^{2}}\left(\sin ^{2} \alpha+\frac{\rho^{2}}{z^{2}}\right)-\frac{\omega_{1}^{2}+\omega_{2}^{2}}{4} T_{0}^{2}\right] \\
& \left.\times \mid I_{m_{0}}\left(2 z \frac{\sin \alpha}{w_{0}^{2}} \rho\right)\right]^{2}\left[\left(\frac{w_{0}^{2}}{2 z c}\right)^{2} \omega_{c}^{2}+\mathrm{i} \frac{w_{0}^{2}}{2 z c} \omega_{d}\right]^{m_{0}} \\
& \times \exp \left[\mathrm{i} \frac{z}{c}\left(1-\frac{\sin ^{2} \alpha}{2}\right) \omega_{d}+\mathrm{i} m_{0}\left(\theta_{1}-\theta_{2}\right)\right] .
\end{aligned}
$$

where the marrow-band approximate: $\omega_{c}^{2} \gg \omega_{d}^{2}$ has been applied. 
Combined with Equations (8), (9), (13) and (14), the SAMCF of output BGLW can be written as $[13,18]$

$$
\begin{aligned}
\left\langle W\left(\rho, \varphi_{1}, \varphi_{2}, z ; t\right)\right\rangle= & E_{0}^{2} \exp \left[-\frac{2 z^{2}}{w_{0}^{2}}\left(\sin ^{2} \alpha+\frac{\rho^{2}}{z^{2}}\right)-\frac{\xi^{2}}{T_{0}^{2}}\right] \\
& \times\left|I_{m_{0}}\left(2 z \frac{\sin \alpha}{w_{0}^{2}} \rho\right)\right|^{2} \times \sum_{q=0}^{m_{0}} \frac{m_{0} !}{\left(m_{0}-q\right) ! q !} 2^{q-1} \\
& \times\left(\frac{w_{0}^{2}}{2 z c T_{0}}\right)^{2 m_{0}-q}\left(2 m_{0}-2 q-1\right) ! ! D_{q}\left(2 \frac{\xi}{T_{0}}\right) \\
& \times \exp \left\{-\frac{2 \rho^{2}}{\rho_{o c \zeta}^{2}}\left[1-\cos \left(\varphi_{1}-\varphi_{2}\right)\right]+\operatorname{i} m_{0}\left(\varphi_{1}-\varphi_{2}\right)\right\} .
\end{aligned}
$$

where $\xi=t-z / c\left[1-\sin ^{2} \alpha / 2\right], D_{q}($.$) denotes parabolic cylinder function, and N ! !$ means taking the double factorial of $N$.

\subsection{Received Probability of Signal Vortex Modes}

Using the plane harmonic superposition theory of complex light waves, we can express the BGLW $u_{p}(\rho, \theta, z, \omega)$ in Equation (5) as [27]

$$
u_{p}(\rho, \theta, z, \omega)=\frac{1}{\sqrt{2 \pi}} \sum_{m} A_{m}(\rho, z, t) \exp (\mathrm{i} m \theta)
$$

where $m$ is the topological charge number of the vortex mode in turbulent seawater. By comparing Equation (5) and Equation (16), we can obtain the superposition coefficient $A_{m}(\rho, z, t)$, that is

$$
\begin{aligned}
A_{m}(\rho, z, t) & =\frac{1}{\sqrt{2 \pi}} \int_{0}^{2 \pi} u_{p}(\rho, \theta, z, \omega) \exp (-\mathrm{i} m \theta) \mathrm{d} \theta \\
& =\frac{T_{0}}{2 \sqrt{2} \pi} \exp (-\mathrm{i} \widetilde{\Omega} t) \int_{0}^{2 \pi} \int_{-\infty}^{+\infty} \exp \left(-\frac{T_{0}^{2}}{4} \omega^{2}\right) \\
& \times u(\rho, \theta, z, \omega) \exp (-\mathrm{i} \omega t-\mathrm{i} m \theta) \mathrm{d} \omega \mathrm{d} \theta
\end{aligned}
$$

Squaring Equation (17) and performing the turbulence ensemble average, we obtain the received probability density

$$
P\left(m \mid m_{0}\right)=\frac{T_{0}^{2}}{8 \pi^{2}} \iiint \int \Gamma_{2}\left(\rho, \theta_{1}, \theta_{2}, z ; \omega_{1}, \omega_{2}\right) \exp \left[-\mathrm{i}\left(\omega_{1}-\omega_{2}\right) t-\mathrm{i} m\left(\theta_{1}-\theta_{2}\right)\right] \mathrm{d} \theta_{1} \mathrm{~d} \theta_{2} \mathrm{~d} \omega_{1} \mathrm{~d} \omega_{2} .
$$

Further, substituting Equations (9), (11), (13) into Equation (18), we write Equation (18) as

$$
\begin{aligned}
P\left(m \mid m_{0}\right)= & E_{0}^{2} \exp \left[-\frac{2 z^{2}}{w_{0}^{2}}\left(\sin ^{2} \alpha+\frac{\rho^{2}}{z^{2}}\right)-\frac{\xi^{2}}{T_{0}^{2}}\right]\left|I_{m_{0}}\left(2 z \frac{\sin \alpha}{w_{0}^{2}} \rho\right)\right|^{2} \\
& \times \sum_{q=0}^{m_{0}} \frac{m_{0} !}{\left(m_{0}-q\right) ! q !}\left(\frac{w_{0}^{2}}{2 z c T_{0}}\right)^{2 m_{0}-q} 2^{q-1}\left(2 m_{0}-2 q-1\right) ! ! \\
& \times D_{q}\left(\frac{2 \xi}{T_{0}}\right) \iint \mathrm{d} \theta_{1} \mathrm{~d} \theta_{2} \exp \left\{-\frac{2 \rho^{2}}{\rho_{o c \zeta}^{2}}\left[1-\cos \left(\theta_{1}-\theta_{2}\right)\right]\right. \\
& \left.+\mathrm{i}\left(m_{0}-m\right)\left(\theta_{1}-\theta_{2}\right)\right\} .
\end{aligned}
$$


Recalling the integral formula [19]

$$
\int_{0}^{2 \pi} \exp \left[-\mathrm{i} y \theta+x \cos \left(\theta-\theta^{\prime}\right)\right] \mathrm{d} \theta=2 \pi \exp \left(-\mathrm{i} y \theta^{\prime}\right) I_{y}(x),
$$

we have

$$
\begin{aligned}
P\left(m \mid m_{0}\right)= & \pi E_{0}^{2} \exp \left[-2 \frac{z^{2}}{w_{0}^{2}} \sin ^{2} \alpha-\frac{\xi^{2}}{T_{0}^{2}}-2\left(\frac{1}{w_{0}^{2}}+\frac{1}{\rho_{o c \zeta}^{2}}\right) \rho^{2}\right] \\
& \times I_{m-m_{0}}\left(2 \frac{\rho^{2}}{\rho_{o c \zeta}^{2}}\right)\left|I_{m_{0}}\left(2 z \rho \frac{\sin \alpha}{w_{0}^{2}}\right)\right|^{2} \\
& \times \sum_{q=0}^{m_{0}} \frac{m_{0} !}{\left(m_{0}-q\right) ! q !} 2^{q}\left(\frac{w_{0}^{2}}{2 z c T_{0}}\right)^{2 m_{0}-q} \\
& \times\left(2 m_{0}-2 q-1\right) ! ! D_{q}\left(2 \frac{\xi}{T_{0}}\right) .
\end{aligned}
$$

By integrating Equation (21) over the receiving aperture $D$, we have the received probability of vortex modes of the BGLW in seawater turbulence

$$
P(m)=P_{D}\left(m \mid m_{0}\right) / \sum_{m=-\infty}^{\infty} P_{D}\left(m \mid m_{0}\right)
$$

and

$$
P_{D}\left(m \mid m_{0}\right)=2 \pi \int_{0}^{D / 2} P\left(m \mid m_{0}\right) \rho \mathrm{d} \rho,
$$

where $m=m_{0}$ means the received probability of signal vortex modes. But, $m \neq m_{0}$ implies the received probability of crosstalk vortex modes.

\section{Discussion}

Now, we begin to comprehensively analyze the influence of the beam parameters and turbulent parameters on the transmission of vortex modes carried by BGLW. In the following discussion, unless otherwise specified, we set the parameters: $z=200 \mathrm{~m}, m=1, m_{0}=1, w_{0}=0.1 \mathrm{~m}, D=0.3 \mathrm{~m}$, $\alpha=0.001, \omega=-4.5, T_{0}=20 \mathrm{fs}, \zeta=2, \varepsilon=10^{-6} \mathrm{~m}^{2} / \mathrm{s}^{3}, \eta=0.001 \mathrm{~m}$, and $\chi_{T}=10^{-8} \mathrm{~K}^{2} / \mathrm{s}$. The evolution curve of the spectrum average coherence length of spherical wave has been given by Figure 1a,b. Next, Figure 2 will display the influence of beam parameters and channel parameters on signal vortex mode transmission; Figures 3-5 will display the influence of turbulence parameters on signal vortex mode transmission; Figures 6 and 7 will display the influence of beam parameters on signal vortex mode transmission.

Figure 2a gives the received probability of signal vortex modes $\left(\left|m-m_{0}=0\right|\right)$ under different co-moving coordinate $\xi$ and topological charge number $m_{0}$, and Figure $2 \mathrm{~b}$ gives the received probability of signal vortex modes under different propagation distance $z$ and topological charge number $m_{0}$. Figure 2a shows that increasing the topological charge number $m_{0}$ will reduce the received probability of signal vortex modes. But, what surprises us is when topological charge number of the vortex mode increases from 0 to 6 , the received probability decreases less than 0.016 which is far less than the Laguerre-Gaussian beam [1]. And Figure $2 b$ clearly shows that the received probability is still above 0.75 even the propagation distance $z$ reaches $500 \mathrm{~m}$. These results once again indicate that, in turbulent seawater, the transmission of the vortex mode carried by the BGLW is better than that of the vortex mode carried by Laguerre-Gaussian beam. 


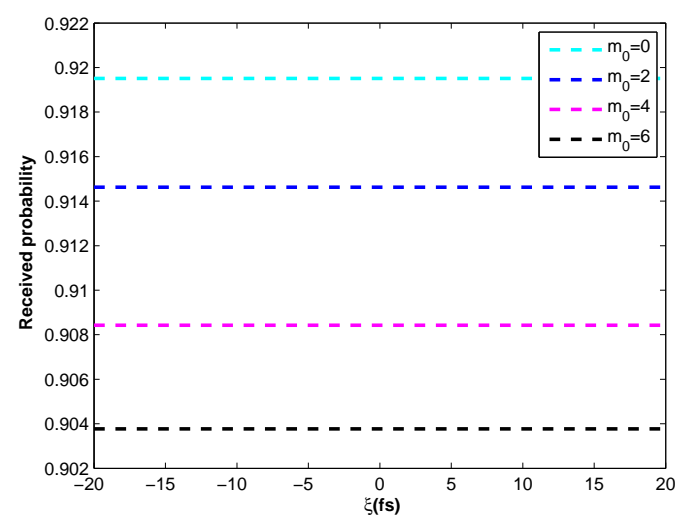

(a)

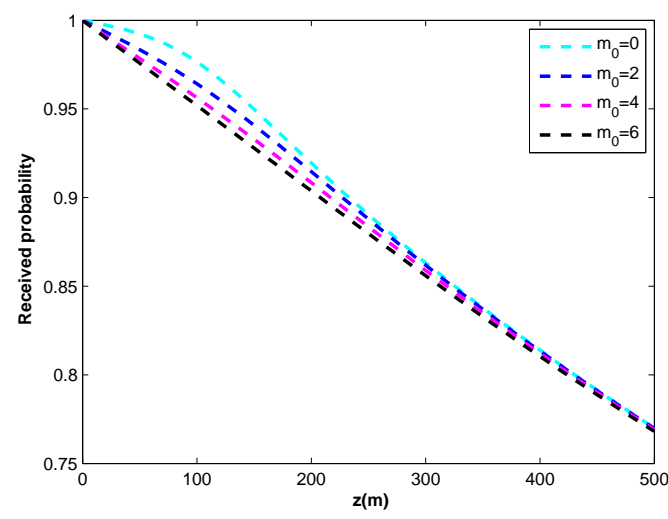

(b)

Figure 2. Received probability of the vortex modes of localized wave of Bessel-Gaussian amplitude envelope under different co-moving coordinate $\xi$ and topological charge number $m_{0}$ in (a) and Received probability of the vortex modes of localized wave of Bessel-Gaussian amplitude envelope under different propagation distance $z$ and topological charge number $m_{0}$ in (b).

To further research the effects of the temperature fluctuation strength of seawater turbulence, we plot the received probability of signal vortex modes under different "temperature structure" constant $C_{m}^{2}$ and anisotropic factor $\zeta$ in Figure 3. We can see that as $C_{m}^{2}$ changes from $10^{-18} \mathrm{~m}^{-2 / 3}$ to $10^{-14} \mathrm{~m}^{-2 / 3}$, the received probability of the signal vortex modes is always decreasing. When $C_{m}^{2}$ is closer to $10^{-18} \mathrm{~m}^{-2 / 3}$, i.e., weak turbulence, turbulence has little disturbance for the vortex mode. This is because that beam wander can be neglected in very weak turbulence. When $C_{m}^{2}$ is closer to $10^{-14} \mathrm{~m}^{-2 / 3}$, i.e., strong turbulence, turbulence severely disturbing with signal propagation. Figure 3 also shows larger anisotropic factor $\zeta$ is conducive to the transiting of signal vortex modes.

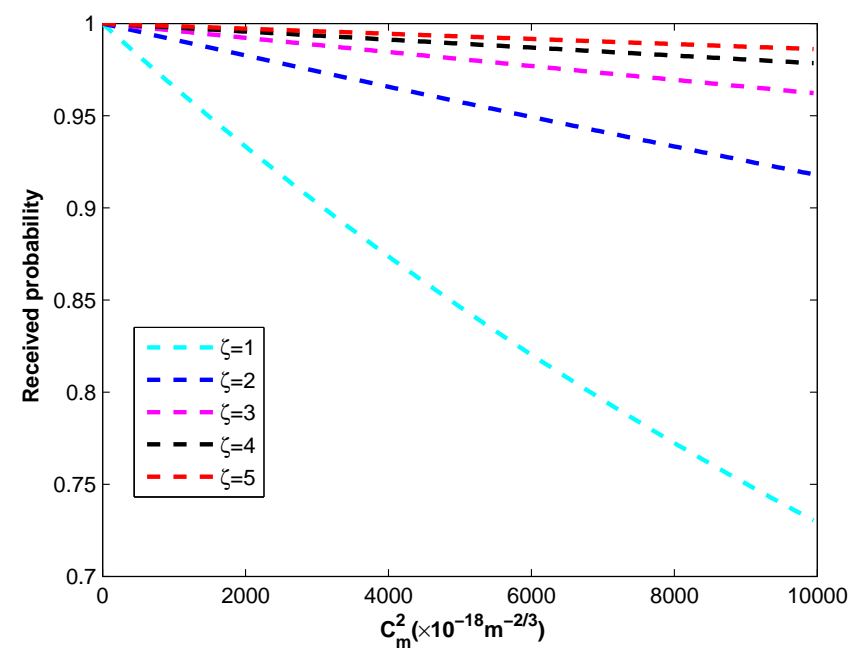

Figure 3. Received probability of the vortex modes of localized wave of Bessel-Gaussian amplitude envelope in seawater turbulence under different "temperature structure" constant $C_{m}^{2}$ and anisotropic factor $\zeta$.

To explore the effects of turbulent parameters on the received probability of vortex modes of the BGLW, in Figure 4, we plot the curve of the received probability as the function of the ratio of temperature and salinity $\omega$ and the inner scale $\eta$. We can see that the received probability of signal vortex modes increases with the increase of inner scale $\eta$ or the decrease of $\omega$. The former is consistent with the theory that the larger the inner scale of the turbulent eddy is, the smaller the scintillation will 
be, and the smaller the disturbance to the signal vortex modes will be. As for the latter, when $\omega$ is close to 0 , salinity dominates heavily, and the curve goes down dramatically, which means that the vortex mode is more sensitive to salinity fluctuations than to temperature fluctuations in turbulence.

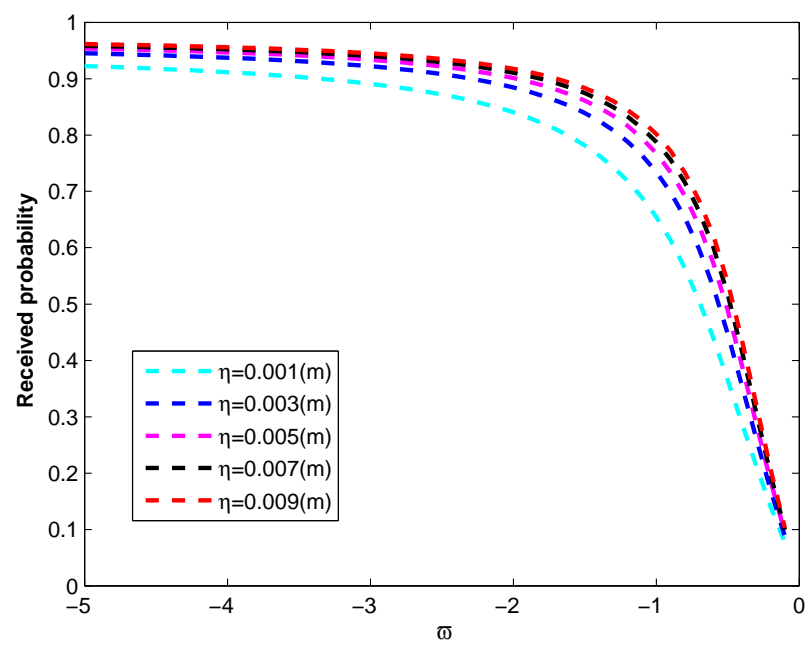

Figure 4. Received probability of the vortex modes of localized wave of Bessel-Gaussian amplitude envelope in seawater turbulence under different ratio of temperature and salinity $\omega$ and inner scale $\eta$.

To further understand the impact of seawater on the received probability of vortex modes of the BGLW, we plot the dissipation rate of the mean-squared temperature $\chi_{T}$ and the dissipation rate of kinetic energy per unit mass $\varepsilon$ of fluid in Figure 5. Figure 5 reveals that the received probability of signal vortex modes increases with the increase of $\varepsilon$ or the decrease of $\chi_{T}$, which can be explained that the larger $\varepsilon$ or the smaller $\chi_{T}$ mean a weaker seawater turbulence, so beam has smaller wander. More significantly, Figure 5 shows that $\chi_{T}$ affects vortex modes transmission much more than $\varepsilon$ does.

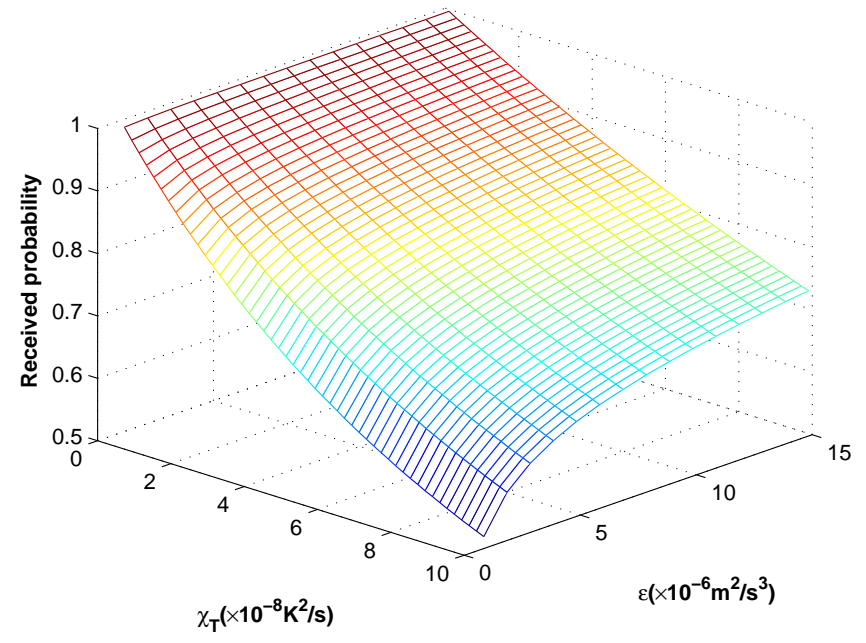

Figure 5. Received probability of the vortex modes of localized wave of Bessel-Gaussian amplitude envelope in seawater turbulence under different dissipation rate of the mean-squared temperature $\chi_{T}$ and dissipation rate of kinetic energy per unit mass of fluid $\varepsilon$.

Also, to understand the influence of beam waist and receiving aperture size on the received probability of signal vortex modes, we show the evolution curve of the received probability of signal vortex modes with the change of beam waist $w_{0}$ and receiving aperture diameter $D$ in Figure 6 . Figure 6 shows that with the increase of the beam waist $w_{0}$, the received probability of signal vortex modes 
keeps increasing until saturation, and a maximum effective waist $w_{0 b}$ exists before the saturated zone. Furthermore, the received probability of signal vortex modes increases with the decrease of the receiver aperture size $D$. The rationality of each numerical curve in Figure 6 can be explained by that the optical path difference among rays passing through turbulence within the range of the received beam diameter varies with the increase or decrease of the size of the beam waist and the receiving aperture. For the waist effect, when the beam waist is smaller than the outer scale of turbulence, with the increase of the beam waist, the beam wander generated by turbulence decreases, the optical path difference between various rays in the receiving aperture decreases, and the wavefront distortion of the transmitted beam caused by turbulence disturbance is reduced, so as to improve the received probability of signal vortex modes. However, when the beam waist is larger than or equal to the outer scale of turbulence, with the increase of the beam waist, the beam wander generated by turbulence is still decreasing. However, rays in the receiving aperture pass through turbulence with different refractive index. Thus, the reduction of optical path difference caused by the decrease of beam wander and the increase of optical path difference caused by the turbulence with different refractive index will be "complementary". It is this kind of "complementarity" that makes the received probability appear at "saturation" or decrease with the further increase of waist. For the receiving aperture size effect, when the aperture is small, the received signal wave is traveling horizontally along the optical axis and passing through the same type of turbulence, and the optical path difference between the rays caused by turbulence is small. However, with the increase of receiving aperture, not only the difference of turbulence passing through but also the difference of geometric path of each ray increases. Thus, the wavefront fluctuation of received optical signal increases, and the received probability of signal vortex modes decreases.

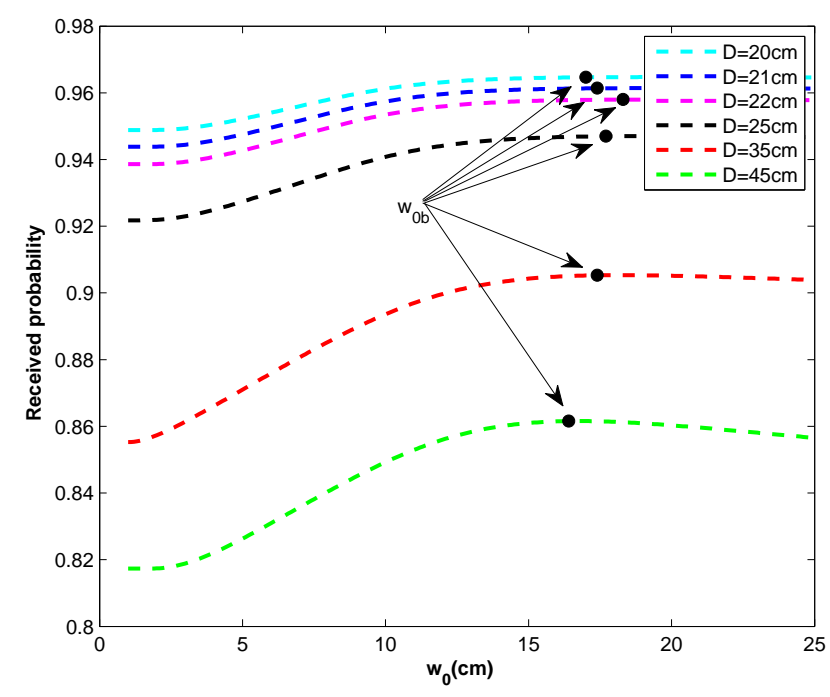

Figure 6. Received probability of the vortex modes of localized wave of Bessel-Gaussian amplitude envelope in seawater turbulence under different beam waist $w_{0}$ and receiving aperture diameter $D$.

Since the Bessel-Gaussian localized wave is a kind of synthetic polychromatic wave, the selection of half-modulated pulse width $T_{0}$ is not negligible. By plotting the received probability under different half-modulated pulse width $T_{0}$ with different Bessel cone angle $\alpha$ in Figure 7, we can see that the received probability of signal vortex modes increases with increasing the half-modulated pulse width $T_{0}$. This result is consistent with the law of Figure $1 \mathrm{~b}$ and the conclusion that the time average will reduce turbulent scintillation [21]. If the Bessel cone angle $\alpha$ increases, the received probability decreases. This result is because that the small Bessel cone angle $\alpha$ means the long collimation distance, resulting in weak wavefront distortion, while weak wavefront distortion leads to low vortex crosstalk. In other words, we had better select the small Bessel cone angle $\alpha$ and large half-modulated pulse 
width $T_{0}$ to weaken the vortex crosstalk. These conclusions are good news for the application of long-distance submarine wireless optical communication based on vortex mode.

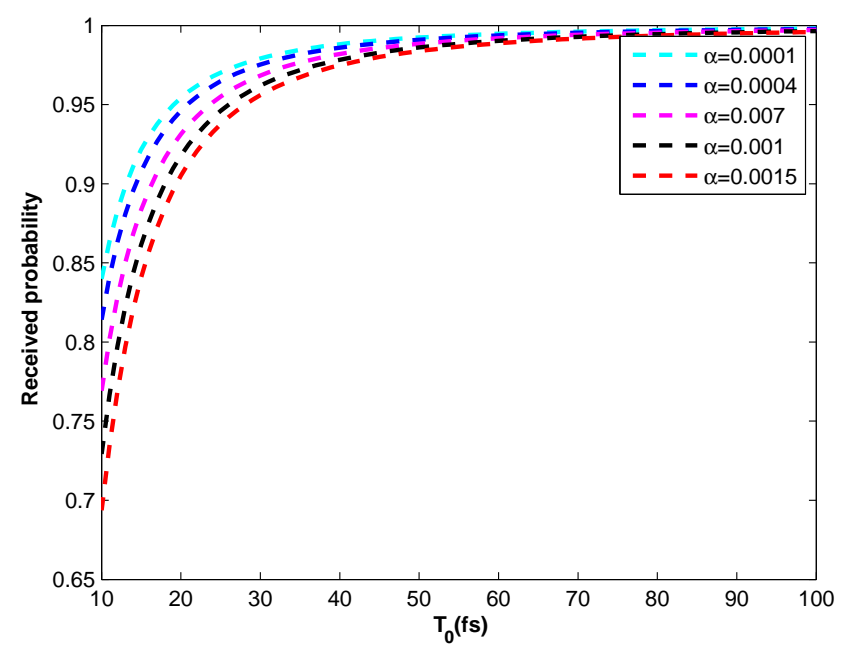

Figure 7. Received probability of the vortex modes of localized wave of Bessel-Gaussian amplitude envelope in seawater turbulence under different half-modulated pulse width $T_{0}$ and Bessel cone angle $\alpha$.

\section{Conclusions}

In order to explore the use of vortex modes to improve the channel capacity of information transmitted by underwater optical communication system, we focus on the transiting of vortex modes carried by the BGLW in turbulent seawater. In this paper, we derive the spectrum average coherence length of spherical wave, the SAMCF of BGLW, and establish the received probability of vortex modes. Our paper indicates that BGLW is a kind of vortex beam which is more promising than other vortex waves to improve transmission of vortex modes. For the given receiver aperture and turbulence parameters, we can utilize larger half-modulated pulse width, smaller Bessel diffraction angle and the maximum effective beam waist to improve the transmission performance of vortex modes. Our paper indicates that we can utilize the BGLW with vortex mode as a communication carrier to increase the amount of communication data transmission in the system of remote submersibles, submarines, analysis of marine resources, and exploration of undersea gas and oil.

Author Contributions: Methodology, Software and Writing, S.D. and Y.Z. (Yixin Zhang); Formal Analysis, Y.Z. (Yixin Zhang); Writing-Original Draft Preparation, Y.Z. (Yun Zhu).

Funding: This research was funded by the National Natural Science Foundation of China (NSFC) [Grant Nos. 61701196, 61871202].

Conflicts of Interest: The authors declare no conflict of interest.

\section{References}

1. Paterson, C. Atmospheric turbulence and orbital angular momentum of single photons for optical communication. Phys. Rev. Lett. 2005, 94, 153901. [CrossRef] [PubMed]

2. Wei, M.; Wang, J.; Zhang, Y.; Hu, Z. Orbital-angular-momentum photons for optical communication in non-Kolmogorov atmospheric turbulence. Opt. Commun. 2018, 10, 89-93. [CrossRef]

3. Wei, M.; Wang, J.; Zhang, Y.; Hu, Z. Universal decay of quantumness for photonic qubits carrying orbital angular momentum through atmospheric Turbulence. IEEE Photonics J. 2018, 10, 1-9. [CrossRef]

4. Zhang, Y.; Shan, L.; Li, Y.; Yu, L. Effects of moderate to strong turbulence on the Hankel-Bessel-Gaussian pulse beam with orbital angular momentum in the marine atmosphere. Opt. Express 2017, 25, 33469-33479. [CrossRef] 
5. Lu, W.; Liu, L.; Sun, J.F. Influence of temperature and salinity fluctuations on propagation behaviour of partially coherent beams in oceanic turbulence. J. Opt. A Pure Appl. Opt. 2006, 8, 1052-1058. [CrossRef]

6. Wu, C.; Ko, J.; Rzasa, J.R.; Paulson, D.A.; Davis, C.C. Phase and amplitude beam shaping with two deformable mirrors implementing input plane and Fourier plane phase modifications. Appl. Opt. 2018, 57, 2337-2345. [CrossRef] [PubMed]

7. Fried, D.L. Branch point problem in adaptive optics. J. Opt. Soc. Am. A 1998, 15, 2759-2768. [CrossRef]

8. Huang, Y.; Zhang, B.; Gao, Z.; Zhao, G.; Duan, Z. Evolution behavior of Gaussian Schell-model vortex beams propagating through oceanic turbulence. Opt. Express 2014, 22, 17723-17734. [CrossRef]

9. Liu, D.; Wang, Y.; Yin, H. Evolution properties of partially coherent flat-topped vortex hollow beam in oceanic turbulence. Appl. Opt. 2015, 54, 10510-10516. [CrossRef]

10. Zhao, Y.; Xu, J.; Wang, A.; Lv, W.; Zhu, L.; Li, S.; Wang, J. Demonstration of data-carrying orbital angular momentum-based underwater wireless optical multicasting link. Opt. Express 2017, 25, 28743-28751. [CrossRef]

11. Liu, D.; Wang, Y.; Wang, G.; Luo, X.; Yin, H. Propagation properties of partially coherent four-petal Gaussian vortex beams in oceanic turbulence. Laser Phys. 2017, 27, 016001. [CrossRef]

12. $\mathrm{Xu}, \mathrm{J}$.; Zhao, D. Propagation of a stochastic electromagnetic vortex beam in the oceanic turbulence. Opt. Laser Technol. 2014, 57, 189-193. [CrossRef]

13. Li, Y.; Yu, L.; Zhang, Y. Influence of anisotropic turbulence on the orbital angular momentum modes of Hermite-Gaussian vortex beam in the ocean. Opt. Express 2017, 25, 12203-12215. [CrossRef] [PubMed]

14. Willner, A.E.; Zhao, Z.; Ren, Y.; Li, L.; Xie, G.; Song, H.; Liu, C.; Zhang, R.; Bao, C.; Pang, K. Underwater optical communications using orbital angular momentum-based spatial division multiplexing. Opt. Commun. 2018, 408, 21-25. [CrossRef]

15. Hernandez-Figueroa, H.E.; Zamboni-Rached, M.; Recami, E. (Eds.) Localized Wave; Wiley-Interscience, IEEE Press: Hoboken, NJ, USA, 2008.

16. Ciattoni, A.; Conti, C.; Porto, P.D. Vector electromagnetic X waves. Phys. Rev. E 2004, 69, 036608. [CrossRef]

17. Ornigotti, M.; Conti, C.; Szameit, A. Effect of orbital angular momentum on nondiffracting ultrashort optical pulses. Phys. Rev. Lett. 2015, 115, 100401. [CrossRef] [PubMed]

18. Li, Y.; Zhang, Y.; Zhu, Y. Influence of oceanic turbulence on propagation of the Gaussian pulsed X wave carrying orbital angular momentum. Opt. Commun. 2018, 428, 57-62. [CrossRef]

19. Wu, Y.; Zhang, Y.; Li, Y.; Hu, Z. Beam wander of Gaussian-Schell model beams propagating through oceanic turbulence. Opt. Commun. 2016, 371, 59-66. [CrossRef]

20. Andrews, L.C.; Phillips, R.L. Laser Beam Propagation through Random Media; SPIE Press: Washington, DC, USA, 2005.

21. Korotkova, O. Random Light Beams Theory and Applications; CRC Press: Boca Raton, FL, USA, 2013.

22. Gradshteyn, I.S.; Ryzhik, I.M. Table of Integrals, Series and Products, 6th ed.; Academic Press: Beijing, China, 2000.

23. Kelly, D.; Andrews, L.C. Temporal broadening and scintillations of ultrashort optical pulses. Waves Random Media 1999, 9, 307-325. [CrossRef]

24. Karp, S.; Gagliardi, R.M.; Moran, S.E.; Stotts, L.B. Optical Channels; Plenum Press: New York, NY, USA, 1988.

25. Young, C.Y.; Andrews, L.C.; Ishimaru, A. Time-of-arrival fluctuations of a space-time Gaussian pulse in weak optical turbulence: An analytic solution. Appl. Opt. 1998, 37, 7655-7660. [CrossRef]

26. Goodman, J.W. Statistical Optics; John Wiley \& Sons: New York, NY, USA, 2015.

27. Zhu, Y.; Zhang, L.; Hu, Z.; Zhang, Y. Effects of non-Kolmogorov turbulence on the spiral spectrum of Hypergeometric-Gaussian laser beams. Opt. Express 2015, 23, 9137. [CrossRef] [PubMed]

(C) 2019 by the authors. Licensee MDPI, Basel, Switzerland. This article is an open access article distributed under the terms and conditions of the Creative Commons Attribution (CC BY) license (http:// creativecommons.org/licenses/by/4.0/). 